\title{
Extracting Weak PUFs from Differential Nonlinearity of Digital-to-Analog Converters
}

\author{
Andreas Herkle, Holger Mandry, Stefan Reich, Markus Sporer, Joachim Becker, Maurits Ortmanns \\ University of Ulm, Institute of Microelectronics, Ulm, Germany \\ \{andreas.herkle, holger.mandry, stefan.reich, markus.sporer, joachim.becker, maurits.ortmanns\}@uni-ulm.de
}

\begin{abstract}
Physical Unclonable Functions utilize random variations from manufacturing to generate unpredictable, yet repeatable fingerprints of devices for usage in a hardware cryptographic context. Most often, they are dedicated electrical circuits in integrated devices and thus occupy additional space while only few implementations exploiting already existing hardware. In this work, we analyze the possibility to extract hardware unique fingerprints from the distinct differential nonlinearities of analogto-digital converters, which are present in almost every system. The transfer curves from measuring a large set of low-cost analog-to-digital converters with 12 bit resolution are analyzed regarding their quality as system fingerprints. Additional postprocessing methods are investigated for further improvement of the uniqueness metrics. The fingerprints are optimized to a perfect inter-hamming distance of $50 \%$ and close-to-maximum entropy. These improvements come at the cost of a reduced number of extracted bits, yet the minimum achieved number of 440 bits is sufficient for secret key generation. By thresholding and consequently avoiding unstable positions in the extracted bit-strings, the intra-hamming distance of unprocessed transfer curves could be reduced to less than $4 \%$. Further measurements over a large temperature range shows that the error rate due to temperature drift never exceeds $13 \%$.
\end{abstract}

Index Terms-Physical Unclonable Function (PUF), Analog-toDigital Converter (ADC), Differential Nonlinearity (DNL)

\section{INTRODUCTION}

A major increase of internet-connected devices over the last years raised concerns about hardware embedded security. Even the most simple device with insecure communication channels can be a rewarding target for malicious intruders. Although embedded devices tend to have more processing power due to shrinking technology nodes, a trade-off in computing power is always accepted for low-power requirements. For such devices, cryptographic algorithms, systems and primitives are required to be lightweight. Physical Unclonable Functions (PUF) are well-known as such primitives, providing secret, unique and unpredictable hardware fingerprints without the need for power-hungry secure storage. In general, PUFs utilize small random variations from manufacturing to generate a device specific response. A PUF providing a single response only (like a hardware fingerprint) and having no input functionality is called a Weak PUF in opposite to a Strong PUF, which offers an additional challenge-response behavior. The utilized variations come from many sources like random dopant variations and oxide thickness fluctuations and can be measured in many different ways like varying oscillation frequencies [1] or random start-up behavior [2]. Analog-to-digital converters
(ADC) or digital-to-analog converters (DAC) have also been used as the physical sources for PUF key generation. In [3], a DAC-PUF was presented where the correction coefficients of mismatching current sources of a feedback digital-to-analog converter in a Sigma-Delta modulator were used as the source of randomness. Following work measured the mismatch of capacitors in successive approximation register (SAR) ADCs [4], [5], the mismatch of comparators in stochastic ADCs [6] or code deviations in a DAC-ADC chain [7]. This works presents a different approach such that the differential nonlinearities (DNL) of an uncorrected analog-to-digital converter are investigated regarding their PUF metrics.

The rest of this work is organized as follows: Section II presents the measurement method and discusses the extraction of the DNL curves. Section III present different methods for converting these curves to PUF fingerprints and assesses their quality with different common PUF metrics. Section IV concludes this work.

\section{Measurement Setup}

In this work, characterization of ADCs is done by fine granular control of their respective DC inputs. The used ADC is the MCP3201-C by Microchip Technology Inc., which implements a 12 bit conventional SAR conversion of a single pseudo-differential input of up to $5 \mathrm{~V}$. On a PCB, 20 of these low-cost devices are connected by an SPI bus to an STM32F4 micro-controller as the measurement control instance. The input voltage for the ADCs is generated by an external highlinearity DAC, which is the 16 bit PXIe-5451 waveform generator by National Instruments. The extraction of the transfer characteristic of the ADCs is achieved by sweeping their input voltage from $0 \mathrm{~V}$ to $5 \mathrm{~V}$ full-scale, generated by applying a 16 bit input code to the waveform generator ranging from 0 to $2^{16}-1$. Thus, the transfer characteristics, especially the switching points between neighboring conversion steps, can be extracted with a resolution of $1 / 16 \mathrm{LSB}_{\mathrm{ADC}}$, which equals to $76.3 \mu \mathrm{V}$. A total amount of 120 ADC devices was measured $N=100$ times for each input voltage step. A subset of 20 devices was additionally measured in a temperature chamber with a temperature sweep from $-30^{\circ} \mathrm{C}$ to $70^{\circ} \mathrm{C}$ applied.

Fig. 1 shows the mean curves of two measured devices, calculated as $\bar{y}(x)=\frac{1}{N} \cdot \sum_{n=0}^{N} y_{n}(x)$, where $x$ is the input code of the DAC and $y$ is the output code of the ADC undertest. It shows that a single measurement of a transfer curve is prone to switching errors, which occur in the transition zone

C2020 IEEE. Personal use of this material is permitted. Permission from IEEE must be obtained for all other uses, in any current or future media, including reprinting/republishing this material for advertising or promotional purposes, creating new collective works, for resale or redistribution to servers or lists, or reuse of any copyrighted component of this work in other works. 


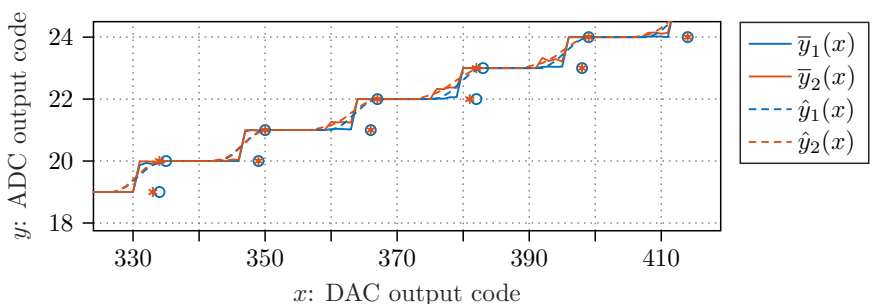

Fig. 1. Exemplary averaged measurement of the transfer curves of two ADC devices and their respective transition zones between two output codes, the final transitions are highlighted with markers

between two ADC levels. In order to get stable transfer curves, either averaging of thousands of samples is required, which is impractical regarding the overall amount of data, or each curve, averaged from fewer samples, has to be smoothed in order to reduce the influence of strong outliers on the overall waveform. A smoothing 5-tap filter with subsequent truncation was applied to calculate the final waveforms as follows:

$$
\begin{aligned}
\hat{y}(x)= & \left\lfloor\frac{1}{15} \cdot(\bar{y}(x+3)+2 \cdot \bar{y}(x+2)+3 \cdot \bar{y}(x+1)\right. \\
& +3 \cdot \bar{y}(x)+3 \cdot \bar{y}(x-1)+2 \cdot \bar{y}(x-2)+\bar{y}(x-3))\rfloor
\end{aligned}
$$

Although the waveforms $\hat{y}(x)$ from Fig. 1 appear to be very similar, they differ in a distinctive way in their transition regions where the flip-over to the next ADC level starts. Both curves in theory have a step width of 16 DAC levels (resolution ratio of $2^{4}$ ). Whenever this is not the case, the respective ADC inherits a differential nonlinearity at that specific level. Such deviations from the ideal transfer curve are random in their position and mostly defined by mismatch of the underlying physical sources used in the analog-to-digital conversion. The differences in the curves are used in the following to extract non-predictable fingerprints.

For a well-known SAR-architecture using a specified number of capacitors, the full curves could be predicted by measuring the capacitors mismatch. Such a predictability was not observed in the measurements and led to the assumption that the ADC might inherit some mismatch scrambling technique. As the internal structure of this specific device is not known, the measurements and the following analysis and post-processing methods are based on the assumption that single values of a measured DNL curve inherit only little to no correlation to other values. This assumption holds true especially for other correlation-free DAC architectures and thus allows a direct transfer of the methods.

Based on the transfer curves, the individual DNLs can be calculated by counting the number of DAC levels, which result in a specific ADC level and then subtracting the expected step width:

$$
D N L(x)=-2^{4}+\sum_{n=0}^{2^{16}-1} \begin{cases}1, & \hat{y}(n)=x \\ 0, & \hat{y}(n) \neq x\end{cases}
$$

Both the DNL curves and their integral nonlinearity (INL) curves of all measured devices are illustrated in Fig. 2. Al-

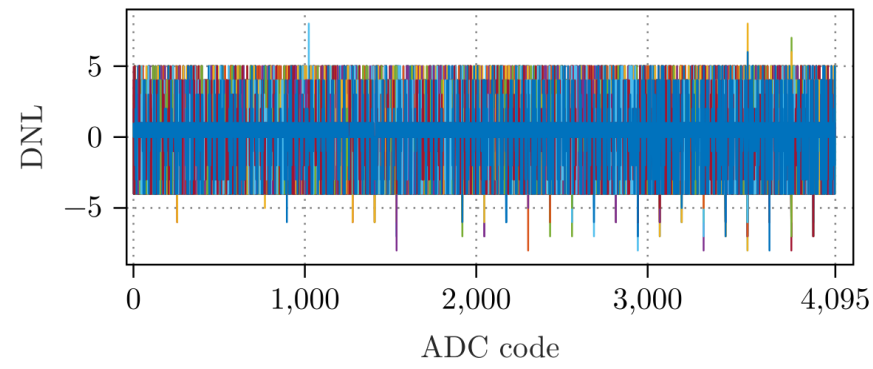

(a)

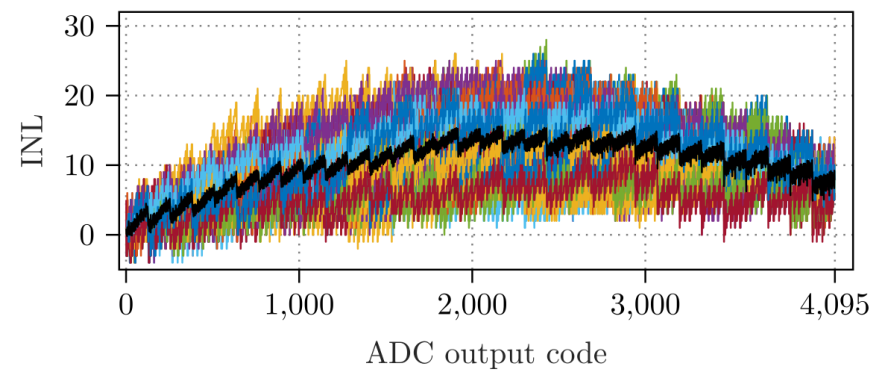

(b)

Fig. 2. (a) DNL curves calculated from all 100 measured 12 bit ADCs, (b) INL curves calculated from the DNL curves with their mean curve hightlighted in black

though the DNL curves appear to be of random nature on first sight, the INL bending into the positive plane only shows that they inherit a bias towards positive values.

\section{Evaluation of Post-Processing Methods}

The extracted DNL curves are of multi-valued nature and range from -8 to +8 . Thus, for a direct usage and evaluation of binary hamming distances, the curves have to be converted to conventional bit-strings. Due to the abundance of zero valued DNLs and the positively skewed non-zero values, this is a nontrivial task. A selection of conversion methods is presented and evaluated regarding their suitability. For the evaluation of the methods, the two standard metrics for PUFs are used: the interhamming distance (inter-HD) as the measure of uniqueness with a desired value close to $50 \%$ and the intra-hamming distance (intra-HD) as the measure of bit stability with a desired value of $0 \%$. For the bit-string $b$ of a device with bit-length $L$ and $M$ readouts, the intra-HD is calculated as

$$
H D_{\text {intra }}(b)=\frac{1}{M-1} \frac{1}{L} \sum_{m=1}^{M-1} \sum_{l=0}^{L-1} \begin{cases}1, & b(m, l) \neq b(0, l) \\ 0, & b(m, l)=b(0, l)\end{cases}
$$

and the inter-HD between two devices $b_{1}$ and $b_{2}$ as

$$
H D_{\text {inter }}\left(b_{1}, b_{2}\right)=\frac{1}{L} \sum_{l=0}^{L-1} \begin{cases}1, & b_{1}(l) \neq b_{2}(l) \\ 0, & b_{1}(l)=b_{2}(l)\end{cases}
$$

Furthermore, the Shannon-entropy is used as an indicator for possible correlations or weaknesses, where a value close to 1 shows perfect randomness. The application of the entropy calculation is applied in two ways: per-bit $\left(H_{b}\right)$ over all devices for the assessment of single bit-positions and per-device $\left(H_{d}\right)$ for the assessment of the average bit-weight, which ideally 
should be close to $50 \%$. For binary alphabets with the bitprobability $p_{i}=p_{i}(l)$ of fixed bit-positions $l, H_{b}(l)$ among all devices $B$ is calculated as

$$
H_{b}(l)=-\sum_{i=0,1} p_{i} \cdot \log _{2}\left(p_{i}\right), p_{i}=\frac{1}{B} \sum_{n=0}^{B-1} \begin{cases}1, & b_{n}(l)=i \\ 0, & b_{n}(l) \neq i\end{cases}
$$

and $H_{d}(b)$ of a single device $b$ among all bit-positions $l$ as

$$
H_{d}(l)=-\sum_{i=0,1} p_{i} \cdot \log _{2}\left(p_{i}\right), p_{i}=\frac{1}{L} \sum_{l=0}^{L-1}\left\{\begin{array}{l}
1, b(l)=i \\
0, b(l) \neq i
\end{array}\right.
$$

\section{A. Conversion Methods and Uniqueness Evaluation}

In the following, different methods for converting the multivalued DNL curves to binary device-unique fingerprints are presented. The methods are analyzed regarding their PUF metrics and their corresponding values are listed in Table I in the upper rows (entropy metrics as percentage of 1).

The first method (bin) converts all non-zero values to binary 1 and all zero values as binary 0 . Due to the imbalance between zero valued and non-zero valued DNLs, the inter-HD is only $34.3 \%$. The per-bit entropy in average is very low with a value of only 0.743 but even worse, with a minimum of 0 .

An alternative method (tern) uses a ternary alphabet, in which negative DNL values are converted to -1 and positive DNL values to +1 while zero values remain untouched. Although the inter-HD sees a slight improvement to $37.3 \%$, the per-bit entropy drops to 0.550 as the amount of zeros are unchanged but the previous probability of a binary 1 is now dispensed to two symbols.

The third method (no-0) first removes all zero valued DNLs and then converts negative DNL values to binary 0 and positive DNL values to binary 1 . As the initial amount of zero valued DNLs had a negative influence, this method provides the first significant improvement with an inter-HD of $48.2 \%$. In addition, the entropy values now have a much larger minimum and both per-bit and per-device entropies are closer to their ideal value of 1 . Still, the previously mentioned bias in the DNL distribution towards positive values limits further improvements when using the DNL values directly.

In order to equalize this distribution, a post-processing technique has to be applied, which in this work was chosen as an adaption of the von Neumann compression $(v N c)$ [8]. Only sign switching transitions in a DNL curve (without zero values in-between) are converted to binary values: transitions from negative to positive values as binary 1 and transitions from positive to negative values as binary 0 . Apart from removing the bias towards positive values, this procedure also scrambles the influence of specific DNL values on the positions $i$ of the converted bit-strings. The resulting inter-HD is at perfect $50 \%$ and the entropy metrics are improved significantly as seen in Table I: $H_{b}$ now holds an average of 0.994 and a minimum of 0.918 and $H_{d}$ is even further improved to an average of 0.997 and a minimum of 0.984 . Although this metrics come with the trade-off that the resulting identifiers are drastically shortened, a minimum length of 440 bits per ADC still provides enough information for generating secret keys.
TABLE I

\begin{tabular}{|c|c|c|c|c|c|c|c|c|}
\hline \multirow{2}{*}{\multicolumn{2}{|c|}{ method }} & \multirow{2}{*}{$\begin{array}{c}\text { inter-HD } \\
\text { avg }\end{array}$} & \multicolumn{2}{|c|}{$H_{b}[\%]$} & \multicolumn{3}{|c|}{$H_{d}[\%]$} & \multirow{2}{*}{$\begin{array}{l}\text { bits } \\
\text { min }\end{array}$} \\
\hline & & & $\min$ avg & $\max$ & $\min$ & avg & $\max$ & \\
\hline \multirow{4}{*}{$\begin{array}{l}\frac{0}{00} \\
: \\
\frac{\omega}{6}\end{array}$} & bin & $34.3 \%$ & $\begin{array}{ll}0 & 74\end{array}$ & 100 & 91 & 94 & 97 & 4093 \\
\hline & tern & $37.3 \%$ & $0 \quad 55$ & 100 & 78 & 82 & 86 & 4093 \\
\hline & no-O & $48.2 \%$ & 5297 & 100 & 96 & 98 & 98 & 1344 \\
\hline & $v N c$ & $50.0 \%$ & 92 & 100 & 98 & 100 & 100 & 440 \\
\hline \multirow{4}{*}{ 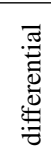 } & bin & $45.7 \%$ & $\begin{array}{ll}52 & 92\end{array}$ & 100 & 95 & 99 & 100 & 4093 \\
\hline & tern & $56.1 \%$ & $44 \quad 85$ & 100 & 84 & 90 & 94 & 4093 \\
\hline & no-0 & $50.0 \%$ & $86 \quad 99$ & 100 & 100 & 100 & 100 & 1525 \\
\hline & $v N c$ & $50.0 \%$ & $84 \quad 99$ & 100 & 99 & 100 & 100 & 616 \\
\hline
\end{tabular}

UNIQUENESS METRICS FOR THE DIFFERENT CONVERSION METHODS.

An alternative approach can be used in a system with multiple ADCs present. By substracting one DNL curve from another, a differential curve can be used as the base of all post-processing methods; their respective metrics are also listed in Table I in the lower rows. Eliminating the bias of single curves with this approach significantly improves the metrics of almost all presented methods by lifting the inter-HD and both entropy metrics closer to their ideal values. Especially the no- $O$ approach outperforms the von Neumann compression in terms of $H_{b}$, which shows that an additional post-processing method on top can also be counter-productive.

\section{B. Evaluation of the Stability with Bit-Error Rates}

The metric of choice for evaluation of the stability of PUF readouts is the intra-Hamming distance (intra-HD). As already illustrated in Fig. 1, the transition regions between two DAC output levels tend to be unstable. In addition, a single error in the DNL measurement affects the width of at least two DNL values and thereby artificially increases the bit-error rates. Thus, the number of samples $N$ chosen for averaging has a crucial influence on the intra-HD.

Fig. 3 a) shows the mean intra-HD of each presented method for an increasing averaging factor $N$ of $\bar{y}(x)$. This increase obviously has a positive effect on the methods bin and tern as they use the full length of the DNL curve and averaging over more samples increases the precision of the DNL calculation. In opposite, all other methods are mostly unaffected with an intra-HD close to $50 \%$ as for these, a single DNL value changing its sign affects the rest of the extracted bit-string such that a previously converted transition is now left out and the whole bit-string is shifted by one position. E.g. the two DNL values $y(x)=-2$ and $y(x+1)=1$ were originally converted to a bit-value of 1 , but for an erroneous measurement of $y(x+1)=0$, the transition from negative to positive is missing and this specific bit-value is then discarded. Thus, error-correction is required after averaging, but its complexity can be reduced as correcting the measured transfer curves or the DNL curves results in much less effort than performing this on the final bit-strings of the different methods.

Because DNL values close to zero are more likely to switch their sign due to erroneous measurements, it seems natural to avoid these values in order to further decrease the intra-HD. 


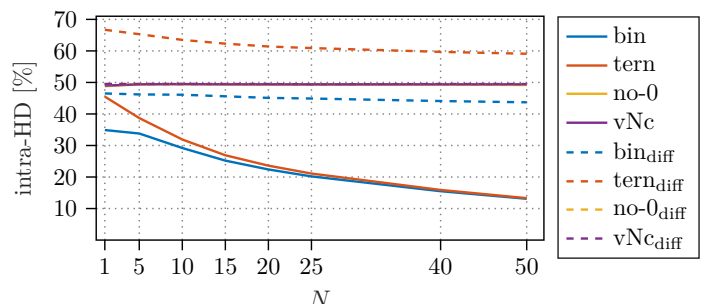

(a)

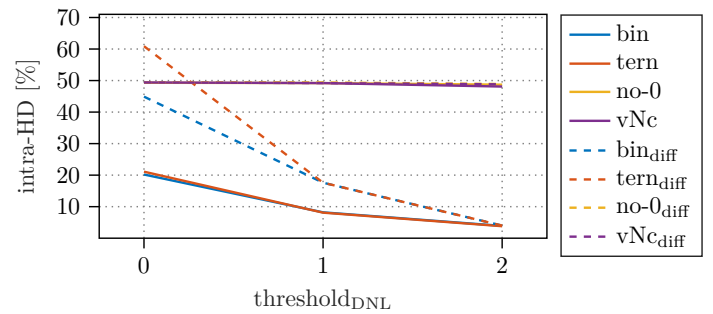

(b)

Fig. 3. (a) Intra-HD in relation to an increasing averaging factor $N$ of the transfer curve, (b) Intra-HD in relation to an increasing DNL value threshold

Such an approach was tested for all methods by introducing an artificial threshold, below which a DNL value was set to zero. Fig. 3 b) shows the intra-HD with an averaging factor of 25 for sweeping the value of this threshold. The stability of the methods bin and tern could be further improved to less then $4 \%$, while the stability of the other methods still suffer from discarded bit-values.

\section{Evaluation of the Temperature Stability}

In addition to the bit-error evaluation, a subset of 20 devices has also been tested for temperature stability. An exemplary readout of a single ADC is plotted in Fig. 4 a), illustrating the measured transfer curve of the whole temperature range from $-30^{\circ} \mathrm{C}$ to $70^{\circ} \mathrm{C}$. Interestingly, the drift of the transfer curve is different for temperatures below and above the nominal temperature of $20^{\circ} \mathrm{C}$. When decreasing the temperature towards $-30^{\circ} \mathrm{C}$, the transition regions between two ADC levels are not shifted in their position but only experience a lift to higher mean values. When the temperature increases towards $70^{\circ} \mathrm{C}$, the transition regions experience the same lift of their mean values but in addition are also shifted to the right.

The effect of this behavior is illustrated in Fig. 4 b) as the mean Euclidean distance between transfer curves of the same device at different temperatures, averaged over all devices. Regarding the dark green line, which illustrates the reference temperature at $20^{\circ} \mathrm{C}$, an increase of up to $6 \%$ can be observed when the temperature only changes by $\pm 10^{\circ} \mathrm{C}$ and increases up to $8.5 \%$ at $70^{\circ} \mathrm{C}$. The colored lines illustrate the respective distances when a different reference is chosen, and as the temperature difference then can be even higher, the Euclidean distances can grow even larger. These differences never grow larger than $13 \%$, and thus a temperature sensor can help correcting the measured transfer curves.

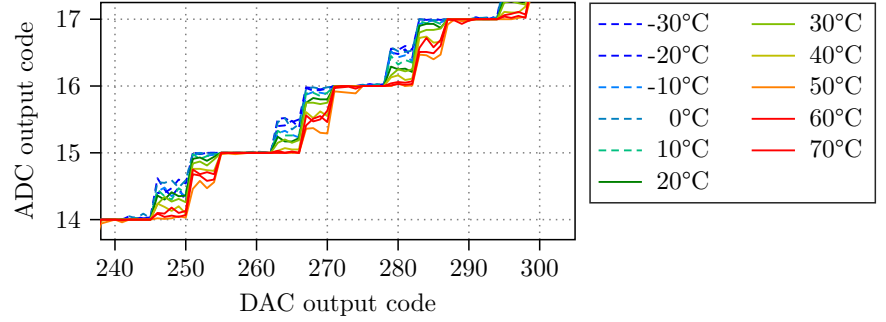

(a)

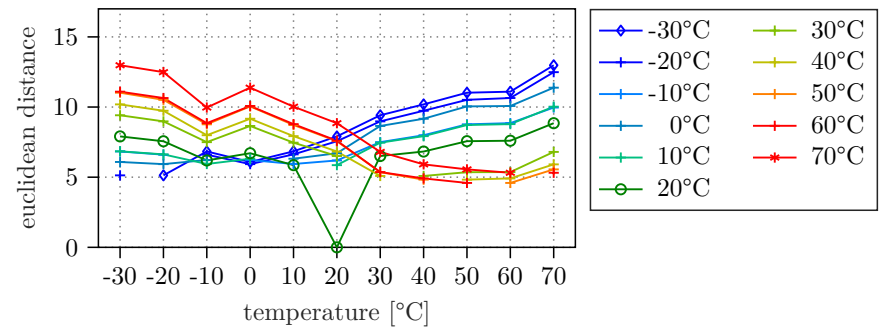

(b)

Fig. 4. (a) Exemplary measurements of the transfer curve of a single device at different temperatures, (b) average Euclidean distance between the transfer curves at different temperatures (self-reference only shown for $20^{\circ} \mathrm{C}$ )

\section{CONCLUSiON}

In this work, DNL and INL of a large set of 12 bit analogto-digital converters were measured by sweeping the input voltage with a 16 bit digital-to-analog converter. The individual differential nonlinearities of each device were calculated and analyzed regarding their potential as device unique fingerprints. Four different post-processing methods were applied to convert the multi-valued curves to bit-strings. An initial approach of directly using the whole bit-string and differentiating between zero and non-zero values resulted in a low interhamming distance of only $34.3 \%$ due to an imbalance between zero and non-zero values. By removing zero valued elements and differentiating between positive and negative values, the inter-hamming distance could be improved to $48.2 \%$. By employing von Neumann compression, the imbalance could be fully removed and thus led to a perfect inter-hamming distance of $50 \%$. The analysis of the readout stability showed that removing zero-valued elements from the initial bit-strings drastically increases the intra-hamming distance of the final outputs. With additional thresholding applied prior to the conversion methods, a minimum intra-hamming distance of $4 \%$ could be achieved. Sweeping the temperature showed that the maximum Euclidean distance between transfer curves is up to $13 \%$. Both results from stability analysis suggest that additional error-correction is inevitable before the application of post-processing methods. But the results show that DNL of ADCs with proper evaluation algorithms can indeed be used as unique hardware fingerprint of PUFs.

\section{ACKNOWLEDGMENT}

This work was funded by the German National Science Foundation DFG under Grant Number FI 982/15-1. 


\section{REFERENCES}

[1] B. Gassend, D. Clarke, M. Van Dijk, and S. Devadas, "Silicon physical random functions," in Proceedings of the 9th ACM conference on Computer and communications security. ACM, 2002, pp. 148-160.

[2] D. E. Holcomb, W. P. Burleson, K. Fu et al., "Initial sram state as a fingerprint and source of true random numbers for rfid tags," in Proceedings of the Conference on RFID Security, vol. 7, no. 2, 2007, p. 01.

[3] A. Herkle, J. Becker, and M. Ortmanns, "Exploiting weak pufs from data converter nonlinearity - e.g., a multibit ct $\triangle$ Emodulator," IEEE Transactions on Circuits and Systems I: Regular Papers, vol. 63, no. 7, pp. 994 1004, July 2016.

[4] Q. Tang, W. H. Choi, L. Everson, K. K. Parhi, and C. H. Kim, "A physical unclonable function based on capacitor mismatch in a chargeredistribution sar-adc," in 2018 IEEE International Symposium on Circuits and Systems (ISCAS), May 2018, pp. 1-5.

[5] A. Shylendra, S. Bhunia, and A. R. Trivedi, "An intrinsic and databasefree authentication by exploiting process variation in back-end capacitors," IEEE Transactions on Very Large Scale Integration (VLSI) Systems, vol. 27, no. 6, pp. 1253-1261, June 2019.

[6] T. Bryant, S. Chowdhury, D. Forte, M. Tehranipoor, and N. Maghari, "A stochastic approach to analog physical unclonable function," in 2016 IEEE 59th International Midwest Symposium on Circuits and Systems (MWSCAS), Oct 2016, pp. 1-4.

[7] A. Duncan, L. Jiang, and M. Swany, "Repurposing soc analog circuitry for additional cots hardware security," in 2018 IEEE International Symposium on Hardware Oriented Security and Trust (HOST), April 2018, pp. 201-204.

[8] S.-H. Kwok, Y.-L. Ee, G. Chew, K. Zheng, K. Khoo, and C.-H. Tan, "A comparison of post-processing techniques for biased random number generators," in Information Security Theory and Practice. Security and Privacy of Mobile Devices in Wireless Communication, C. A. Ardagna and J. Zhou, Eds. Berlin, Heidelberg: Springer Berlin Heidelberg, 2011, pp. 175-190. 